\title{
Validation of an Individualized Measure of Quality of Life, Patient Generated Index, for Use with People with Parkinson's Disease
}

\author{
Ayse Kuspinar $\mathbb{D}^{1},{ }^{1}$ Kedar K. V. Mate $\mathbb{D}^{\mathbb{D}},{ }^{2}$ Anne-Louise Lafontaine, ${ }^{3,4}$ and Nancy Mayo ${ }^{2,5}$ \\ ${ }^{1}$ School of Rehabilitation Science, McMaster University, Hamilton, ON, Canada \\ ${ }^{2}$ Center for Outcomes Research and Evaluation, McGill University Health Centre-Research Institute, Montreal, QC, Canada \\ ${ }^{3}$ Neurology, McGill University Health Centre, Montreal, QC, Canada \\ ${ }^{4}$ Department of Neurology and Neurosurgery, McGill University, Montreal, QC, Canada \\ ${ }^{5}$ School of Physical and Occupational Therapy, McGill University, Montreal, QC, Canada \\ Correspondence should be addressed to Ayse Kuspinar; kuspinaa@mcmaster.ca
}

Received 14 November 2019; Revised 3 February 2020; Accepted 13 February 2020; Published 30 March 2020

Academic Editor: Jeff Bronstein

Copyright (C) 2020 Ayse Kuspinar et al. This is an open access article distributed under the Creative Commons Attribution License, which permits unrestricted use, distribution, and reproduction in any medium, provided the original work is properly cited.

\begin{abstract}
Introduction. Parkinson's disease (PD) affects all aspects of an individual's life and is heterogeneous across people and time. The Patient Generated Index (PGI) is an individualized measure of quality of life (QOL) that allows patients to identify the areas of life that are important to them. Although the PGI has immense potential for use in clinical and research settings, its validity has not been assessed in PD. The purpose of this study is to estimate how well areas of QOL that patients with PD nominate on the PGI agree with ratings obtained from standard outcome measures. Methods. Patients with PD completed the PGI and various standard patient-reported outcome (PRO) measures. The PGI and standard PRO measures were compared at the total score, domain, and item levels. Pearson's correlations and independent $t$-tests were used, as well as positive and negative predictive values. Results. The sample $(n=76)$ had a mean age of 69 (standard deviation 9) and were predominantly men (59\%). The PGI was moderately correlated $(r=-0.35)$ with the standardized disease-specific QOL measure Parkinson's Disease Questionnaire (PDQ- 8 ). Within one severity rating, agreement between the PGI and different standard outcome measures ranged from 85 to $100 \%$ for walking, 69 to $100 \%$ for fatigue, 38 to $75 \%$ for depression, and 20 to $80 \%$ for memory/concentration. Conclusion. This study demonstrates that nominated areas of QOL on the PGI provide comparable results to standard PRO measures, and provides evidence in support of the validity of this individualized measure in PD.
\end{abstract}

\section{Introduction}

Parkinson's disease (PD) affects all aspects of an individual's life and is heterogeneous across people and time. People with PD experience a wide range of symptoms, with some of the most common being limitations in walking [1], fatigue [2], cognitive decline [3], and depression [4].

Assessing quality of life (QOL) has moved to the forefront of clinical research and practice in $\mathrm{PD}[4,5]$. The assessment of QOL is important because it can help identify the aspects of a patient's functioning that are affected by his or her disease and that are potential problems that need to be addressed during treatment. QOL assessments can help prioritize problems, set therapy goals, enhance communication between the clinician and the patient, and help monitor changes or responses to treatment [6].

Standard patient-reported outcome (PRO) measures of QOL generally have predetermined domains and response options (e.g., PDQ-39, SF-36). The underlying assumption with these measures is that (i) the same domains of life are important to all people, and (ii) for each domain, all people have the same needs and goals [7]. Furthermore, these measures assume that there is equal weighting between the domains, or if weights do exist, they are based on values obtained from the general population who have never experienced the disease [8].

Individualized measures of QOL allow patients to identify the domains (or areas of life) that are important to 
them and to assign a weight on the relative importance of each domain. The Patient Generated Index (PGI) is an individualized measure of QOL (IQOL) that allows patients to nominate, rate, and value areas that have the most impact on their quality of life. The validity of the PGI has been tested in other health conditions [9-12], but not yet in PD. Therefore, the objective of this study is to estimate how well areas of QOL identified on the PGI (an individualized measure) agree with ratings obtained from standard PRO measures in people with PD.

\section{Materials and Methods}

2.1. Subjects. Patients with $\mathrm{PD}$ were recruited from the McGill Movement Disorder Clinic in Montreal, Canada. Patients were excluded if they presented with a major comorbid condition (e.g., severe dementia, severe psychiatric, neurological, or other medical condition likely to have major impact on the quality of life, other than PD). During the routine clinical visit, patients were introduced to the study and those interested were presented with a consent form. The protocol was approved by the local Institutional Ethics Review Board.

\subsection{Measures}

\subsubsection{Individualized Measure of Quality of Life}

(1) Patient Generated Index. The Patient Generated Index (PGI) is a short individualized measure of QOL that takes only a couple of minutes to administer [11, 13]. First, participants are asked to identify up to five of the most important areas of their lives affected by PD. Second, they are asked to rate the extent to which they are in the selected areas on a scale of 0 to 10 , where 0 is the worst they can imagine and 10 exactly as they would like to be. In the third phase, patients are given twelve spending "points" to allocate across the areas. The more points a patient spends for an area, the more important that area is. The PGI is calculated as the (sum of (area score $\times$ points spent/12)) [14]. The PGI produces a total score of overall QOL from 0 to 10 , where higher scores indicate better QOL. The PGI has been shown to be reliable, valid, and responsive to change [15].

\subsubsection{Standard Patient-Reported Outcome Measures}

(1) Parkinson's Disease Questionnaire-8. The Parkinson's Disease Questionnaire-8 (PDQ-8) [16] is a self-reported measure of QOL that includes questions on mobility, activities of daily living, emotional well-being, stigma, social support, cognition, communication, and bodily discomfort. Each question is scored from 0 to 4 points and the scores are summed. The summed score is then divided by the total possible score and reported as a percentage out of 100 , where lower scores indicate better quality of life. The PDQ-8 has demonstrated moderate correlations with standard measures of disease severity [16].
(2) RAND-36 Physical Function Index. The Physical Function Index (PFI) of the RAND-36 [17] is composed of 10 questions that describe a range of physical activities including limitations with walking. Each item has 3 response options "yes, limited a lot," "yes, limited a little," and "no, not limited at all." The sum of the responses is taken and scaled from 0 to 100 .

(3) Geriatric Depression Scale. The Geriatric Depression Scale (GDS) [18] is an 8-item self-report measure designed to identify depression in the elderly. Examples of questions in the GDS include "are you basically satisfied with your life?" and "do you feel that your life is empty?". Response options for the 8 items are "yes/no," and one point is assigned to each answer. The measure is scored from 0 to 8 , where higher scores indicate more depression.

(4) Apathy Scale. The Apathy Scale (AS) [19] is an interviewer-administered questionnaire designed to measure loss of motivation, interest, and social engagement. It consists of 14 questions with response options "not at all," "slightly," "some," or "a lot." Scores range from 0 to 42, and higher scores indicate more severe apathy. The AS has demonstrated reliability and validity in PD [19].

(5) Perceived Deficits Questionnaire-20. The Perceived Deficits Questionnaire-20 (PDQ-20) [20] is a self-reported measure of perceived cognitive function. It contains 20 items that assess various domains of cognition: attention, memory, planning, and organization. Response options are in a 5 -point Likert scale, from "never" to "almost always" and rates of " 0 " to " 4 " are given according to the response option selected. The total score is out of 80 , where higher scores indicate greater cognitive impairment. The PDQ-20 has demonstrated good reliability and validity [20].

(6) Visual Analogue Scale (VAS) for Fatigue. Patients were presented with a visual analogue scale from 0 (much fatigue) to 10 (no fatigue) and were asked to mark a line on the scale to indicate how much fatigue they were experiencing. The VAS for fatigue has demonstrated acceptable convergent validity against self-reported measures of fatigue [21].

(7) Visual Analogue Scale (VAS) for Depression. Patients were presented with a visual analogue scale from 0 (much depression) to 10 (no depression) and were asked to mark a line on the scale to indicate what they were experiencing. The VAS for depression has been shown to be significantly correlated with a standardized and lengthier measure of depression (the Beck Depression Inventory) [22].

2.3. Statistical Methods. The PGI and the standard PRO measures were compared at the (1) total score level, (2) domain level, and (3) item level. A domain refers to broad HRQL concepts (e.g., depression), whereas an item refers to a specific question in a PRO measure.

2.3.1. Total Score Level. At the total score level, Pearson's correlation and coefficient of determination were calculated 
to estimate the strength of the association between the PGI and the PDQ-8 (a standard PRO measure of QOL in PD). We hypothesized that the correlation coefficient value between the PGI and the PDQ- 8 would be moderate ( 0.3 to $0.4)[23]$.

2.3.2. Domain Level. At the domain level, the independent $t$-test or the Mann-Whitney $U$ test was calculated, as appropriate, to compare total scores from standard PRO measures that assessed walking (RAND-36 PFI), depression (GDS), and memory/concentration (PDQ-20), between people who nominated and did not nominate those corresponding areas using the PGI. The Shapiro-Wilk test was used to verify the assumption of normality. If the condition of normality was not met, the Mann-Whitney $U$ test was used. We hypothesized that scores on the standard PROs would be statistically significantly different between individuals who nominated and did not nominate a domain.

2.3.3. Item Level. All items on the standard PRO measures were transformed to range from 0 to 10 ( 0 as the worst level and 10 as the best level) to enable direct comparison between them. As the standardized PRO measures had varying numbers of response options ( 3,4 , or 5 ), the PGI severity score (on a scale from 0 to 10 ) was adapted to match these response categories (see Table 1).

Previous studies have demonstrated that, on a scale of $0-10$ (where 0 is none and 10 is severe), a score of 4 or greater for either pain or fatigue is considered a concerning level and requires further comprehensive assessment [24-26]. Thus, since the PGI has a reversed scale (where 0 is severe and 10 is none), the threshold for a concerning score was set at 6 . In other words, a severity score from 0 to 6 on the PGI was defined as concerning, and a score from 7 to 10 was defined as nonconcerning.

At the item level, agreement and positive predictive values (PPVs) and negative predictive values (NPVs) were calculated for walking, fatigue, depression, and memory/ concentration. To estimate PPV and NPV, the score on the standard PRO item represented the true value, and the PGI nominated area was the value to be assessed. The PPV was computed as the number of patients who responded in the concerning range on the standard PRO measure (0 to 6) divided by the total number of patients who nominated that domain on the PGI. A severity score from 0 to 6 out of 10 would be considered concerning, so response categories on standardized PRO measures that fell within this range were also defined as concerning (as shown in Table 1). The NPV was calculated as the number of patients who responded in the nonconcerning range on the standard PRO measure ( 7 to 10) divided by the number of patients who did not nominate that domain on the PGI.

\section{Results}

3.1. Sample Characteristics. Table 2 presents a summary of the sample characteristics. Participants had an average age of 69 (standard deviation: 9.5) and 59\% were men. The average
TABLE 1: Categorization of response options on standard PRO measures and PGI severity rating.

\begin{tabular}{lc}
\hline $\begin{array}{l}\text { Standard patient-reported } \\
\text { outcome measures }\end{array}$ & Patient Generated Index \\
\hline 3 categories & $0-3 ; 4-6 ; 7-10$ \\
4 categories & $0-3 ; 4-6 ; 7-8 ; 9-10$ \\
5 categories & $0-1 ; 2-3 ; 4-6 ; 7-8 ; 9-10$ \\
\hline
\end{tabular}

0 is the worst they can imagine and 10 exactly as they would like to be.

TABle 2: Demographic and clinical characteristics of the sample $(n=76)$.

\begin{tabular}{|c|c|}
\hline Characteristics & Mean (SD) or $N(\%)$ \\
\hline Age (y) & $69.1(9.5)$ \\
\hline Women & $31(41 \%)$ \\
\hline Years since diagnosis & \\
\hline m onset & \\
\hline rigid & $46(71$ \\
\hline f falls (none/rare/monthly) & $41(63) / 16(25$ \\
\hline & \\
\hline $\mathrm{H}$ & \\
\hline Patic & \\
\hline Parkir & \\
\hline Questionnaire-8 (0-100) & $27(14.2)$ \\
\hline RAN & \\
\hline-100$)^{*}$ & \\
\hline Scale $(0-8)^{* *}$ & \\
\hline & \\
\hline$(0-80)^{* * *}$ & $25.8(11.9)$ \\
\hline Apathy Scale $(0-42)^{* * * *}$ & $22.3(3.9)$ \\
\hline \multicolumn{2}{|c|}{$\begin{array}{l}\text { SD, standard deviation; } N \text {, number. }{ }^{*} N=64 ;{ }^{* *} N=70 ;{ }^{* * * * *} N=67 ; \\
\text { QOL. Hoehn and Yahr Stages: } 1-\text { minimal or no functional disability; } \\
\text { Q- symptoms, no impairment of balance; } 3-\text { mild to moderate disability, } \\
\text { still physically independent; } 4-\text { severe disability but still able to walk and } \\
\text { stand unassisted; } 5-\text { confined to bed or wheelchair unless aided. Parkin- } \\
\text { son's Disease Questionnaire- } 8 \text { : lower is better; RAND-36 Physical Function } \\
\text { Index: higher is better; Geriatric Depression Scale: higher is more de- } \\
\text { pression; Perceived Deficits Questionnaire-20: higher is more deficits, } \geq 40 \\
\text { considered cognitively impaired; Apathy Scale: high is more apathy. }\end{array}$} \\
\hline
\end{tabular}

number of years since diagnosis was 6 , and the average number of years since symptom onset was 8 . Participants had an average of 2.3 and median of 2.0 on the Hoehn and Yahr scale. The mean total score for the PGI was 4.2 (standard deviation: 1.8) on a scale from 0 to 10 , where higher scores indicate better quality of life. The average total score for the PDQ-8 was 26.99 (standard deviation: 14.2).

3.2. PGI Responses. The domains generated from the PGI were categorized using the World Health Organization's International Classification of Functioning, Disability, and Health (ICF). The ICF provided a coding framework and common nomenclature for each nominated area. The results of this process have been published previously [27]. The domains reported by patients on the PGI included dexterity, walking, sleep, fatigue, cognition, tremors, sports, depression, anxiety, self-care, speech, socializing, household tasks, bowel and bladder, work/employment, and balance. Figure 1 presents the distribution of the severity rating scores (scaled 


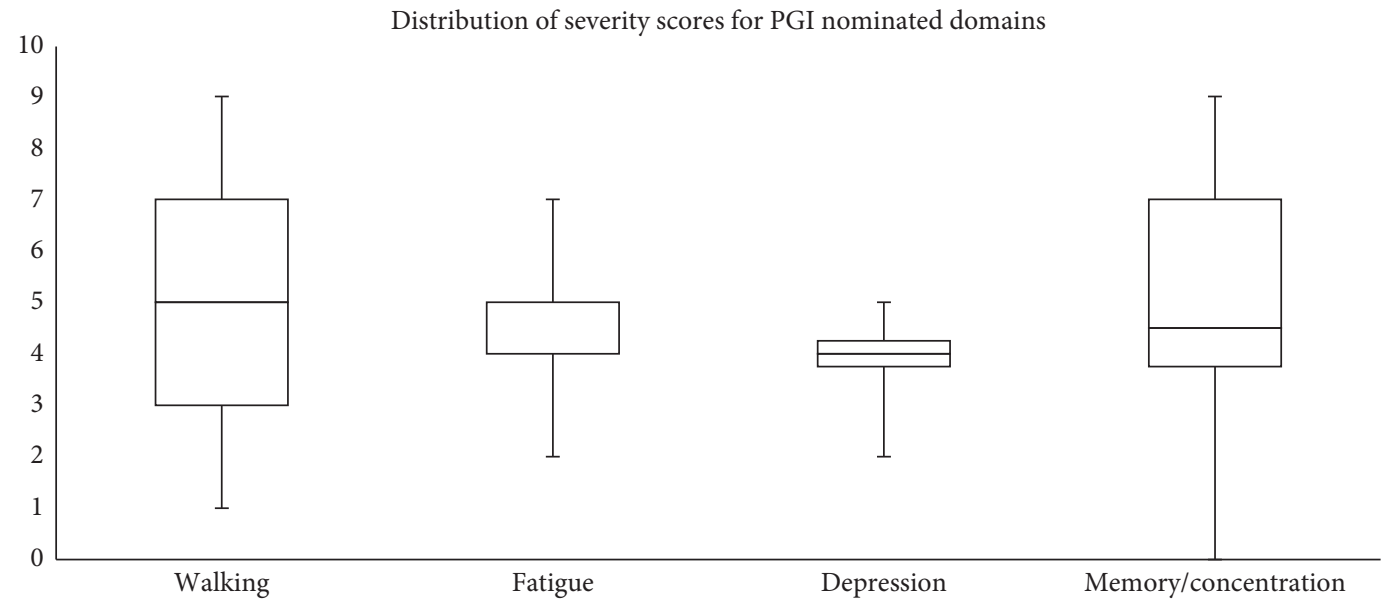

Figure 1: Distribution of the severity scores on a scale from 0 (worst) to 10 (best) for walking, fatigue, depression, and memory/concentration among people who endorsed the area. 0 is the worst they can imagine and 10 exactly as they would like to be.

from 0 to 10) for the PGI domains: walking, depression, and memory/concentration. The borders of the box are the upper $(75 \%)$ and lower (25\%) quartiles, and the horizontal line inside the box is the median. The median severity rating scores for walking, fatigue, depression, and memory/concentration were $5,4,4$, and 4.5 , respectively.

\subsection{Validity Testing}

3.3.1. Total Score Level. Pearson's correlation coefficient between the PGI total score and the PDQ-8, a standard PRO measure of QOL, was $r=-0.35\left(r^{2}=0.12 ; p=0.006\right)$. The distribution of the PDQ-8 and the PGI is provided in the Supplementary Materials (Figures 2 and 3).

3.3.2. Domain Level. Table 3 presents the results of an independent $t$-test or Mann-Whitney $U$ test for participants who nominated walking, depression, and memory/concentration on the PGI, in comparison with participants who did not select these areas. The independent $t$-test was used for the PDQ-20. The Mann-Whitney U test was used for the RAND-36 PFI and the GDS because the assumptions of normality were not met. Participants who nominated walking, depression, and memory/concentration on the PGI had significantly worse scores on the related standard PRO measure than participants who did not nominate these domains. For example, the median score on the RAND-36 PFI was significantly lower $(p=0.0006)$ for participants who nominated walking as an area of concern (40.0) compared to participants who did not nominate walking (80.0). Similar results were observed on the GDS for depression and the PDQ-20 for cognition.

3.3.3. Item Level. Table 4 presents the extent to which severity ratings on the PGI agreed with the severity rating on related standard PRO measures. The table shows the results for the percent agreement within one severity level, and the PPV and NPV for detecting a concerning level of walking, fatigue, depression, or memory/concentration on standard PRO measures.
There were three items related to walking from the RAND-36 PFI (walking one block, walking several blocks, and walking more than a kilometer). Percent agreement within one severity level ranged from $85 \%$ to $100 \%$ for these items. If walking was nominated on the PGI, the PPV for a concerning score across the three standard PRO items in the RAND-36 PFI ranged from $55 \%$ to $80 \%$; if walking was not nominated, the NPV ranged from $59 \%$ to $80 \%$.

For fatigue, the PGI severity rating was compared against one item from the AS (energy for daily activities) and one item from a fatigue-specific VAS (much fatigue to no fatigue). Percent agreement between the PGI severity rating and the standard PRO items was 69\% (energy for daily activities) and $100 \%$ (VAS - much fatigue to no fatigue). The PPV for a concerning score across standard PROs was between $69 \%$ and $94 \%$ when fatigue was nominated, and the NPV was lower at $33 \%$ and $41 \%$ when fatigue was not nominated.

For depression, the PGI was compared against one item that was a depression-specific VAS (much depressed to no depression) and one item from the PDQ-8 (felt depressed). Percent agreement within one severity level was 38\% (PDQ8-felt depressed) and 75\% (VAS - much depression to no depression). The PPV was between $85 \%$ and $86 \%$, and the NPV was between $63 \%$ and $66 \%$.

For memory/concentration, the PGI was compared against 20 items from the PDQ-20. Percent agreement between the PGI and the PDQ-20 items ranged from $20 \%$ to $80 \%$. Seven out of 20 items demonstrated $\geq 70 \%$ agreement between the PGI and the standard PRO. Eleven out of 20 items had percent agreement values between $50 \%$ and $69 \%$. Only two items had percent agreement values less than $50 \%$. PPV values ranged from $27 \%$ to $93 \%$ and the NPV values ranged from $37 \%$ to $92 \%$. Table 4 presents the results for the cognition items that demonstrated the highest percent agreement $(\geq 70 \%)$.

\section{Discussion}

This study evaluated the construct validity of an individualized measure of QOL, the PGI, in people with PD. The 
TABLe 3: Comparison of the PGI against standard PRO measures at the domain level among people who endorsed the area.

\begin{tabular}{|c|c|c|c|}
\hline & $\begin{array}{l}\text { Identified as an area of } \\
\text { concern on the PGI } \\
\text { Mean (SD) } \\
\text { Median }(Q 1-Q 3)(N)\end{array}$ & $\begin{array}{l}\text { Not identified as an area of } \\
\text { concern on the PGI } \\
\text { Mean (SD) } \\
\text { Median }(Q 1-Q 3)(N)\end{array}$ & $\begin{array}{c}\text { Independent } t \text {-test or } \\
\text { Mann-Whitney } U \text { test, } p \text { value }\end{array}$ \\
\hline $\begin{array}{l}\text { Walking (higher is better) } \\
\text { RAND-36 physical function index }\end{array}$ & $\begin{array}{c}45.0(27.3) \\
40.0(25.0-65.0)(19) \\
\end{array}$ & $\begin{array}{c}71.9(24.0) \\
80.0(50.0-95.0)(46) \\
\end{array}$ & 0.0006 \\
\hline $\begin{array}{l}\text { Depression (higher is worse) } \\
\text { Geriatric depression scale }\end{array}$ & $\begin{array}{c}4.5(2.8) \\
4.5(2.5-7.0)(8) \\
\end{array}$ & $\begin{array}{c}1.8(2.2) \\
1.0(0.0-3.0)(62) \\
\end{array}$ & 0.009 \\
\hline $\begin{array}{l}\text { Memory/concentration (higher is worse) } \\
\text { Perceived Deficits Questionnaire- } 20\end{array}$ & $\begin{array}{c}34.6(12.1) \\
36.5(25.0-44.0)(15)\end{array}$ & $\begin{array}{c}23.4(10.6) \\
22.0(17.0-30.0)(52)\end{array}$ & 0.0004 \\
\hline
\end{tabular}

SD, standard deviation; $N$, number; $Q 1$, quartile 1; Q3, quartile 3.

TABle 4: Comparison of the PGI against standard PRO measures at the item level among people who endorsed the area.

\begin{tabular}{|c|c|c|c|c|c|}
\hline Items & $\begin{array}{l}\text { Number of } \\
\text { response options }\end{array}$ & $\begin{array}{l}\text { Standard PRO } \\
\text { measure }\end{array}$ & $\begin{array}{c}\text { Agreement } \\
\Delta \pm 1 \\
N(\%)\end{array}$ & PPV (\%) & NPV (\%) \\
\hline \multicolumn{6}{|l|}{ Walking } \\
\hline Walking several blocks & 3 & RAND-36 PFI & $20(100)$ & 70 & 63 \\
\hline Walking one block & 3 & RAND-36 PFI & $18(90)$ & 55 & 80 \\
\hline Walking more than a kilometer & 3 & RAND-36 PFI & $17(85)$ & 80 & 59 \\
\hline \multicolumn{6}{|l|}{ Fatigue } \\
\hline Energy for daily activities & 4 & AS & $16(100)$ & 69 & 41 \\
\hline Much fatigue to none & 10 & VAS & $11(69)$ & 94 & 33 \\
\hline \multicolumn{6}{|l|}{ Depression } \\
\hline Much depression to no depression & 10 & VAS & $6(75)$ & 86 & 63 \\
\hline Felt depressed & 5 & PDQ-8 & $3(38)$ & 85 & 66 \\
\hline \multicolumn{6}{|l|}{ Memory/concentration } \\
\hline Forget if you had already done something & 5 & PDQ-20 & $12(80)$ & 80 & 75 \\
\hline Forget what you came into the room for & 5 & PDQ-20 & $11(73)$ & 67 & 46 \\
\hline Trouble concentrating & 5 & PDQ-20 & $11(73)$ & 53 & 70 \\
\hline Forget what you did the night before & 5 & PDQ-20 & $11(73)$ & 53 & 70 \\
\hline Find your mind drifting & 5 & PDQ-20 & $11(73)$ & 67 & 56 \\
\hline Forget what you did last weekend & 5 & PDQ-20 & $11(73)$ & 67 & 62 \\
\hline Forget to take your medication & 5 & PDQ-20 & $11(73)$ & 67 & 74 \\
\hline
\end{tabular}

$N$, number; PPV, positive predictive value; NPV, negative predictive value; PFI, Physical Function Index; AS, Apathy Scale; VAS, visual analogue scale; PDQ20, Perceived Deficits Questionnaire-20.

results provided support for the validity of the scores produced by the PGI in comparison with standard PRO measures.

Patients who nominated walking, fatigue, depression, and memory/concentration as an area of concern (regardless of whether it was rated poorly or not) scored significantly lower on corresponding standard PRO measures, thus supporting construct validity of the PGI. Furthermore, agreement between PGI severity ratings and corresponding standard PRO items were relatively high for walking and fatigue. For depression, there was high agreement with the VAS but not with the depression item from the PDQ-8 (how often during the last month have you felt depressed? Never, Occasionally, Sometimes, Often, and Always). The VAS scale might have performed better because it has a 10-point scale similar to the PGI. For cognition, the PGI demonstrated moderate to high agreement with most of the items from the PDQ-20, with the exception of two. These two items were in relation to planning and organization, which were not nominated as an area of concern by our sample.

The PGI was moderately correlated with the PDQ-8, a standard QOL measure developed for people with PD. These results were in line with our hypothesis, as previous research has demonstrated moderate associations between IQOL measures and standard measures of QOL (e.g., subscales of the SF-36) [28]. Strong correlations are typically not observed because the domains nominated by patients can be different from what are included in standard measures developed by researchers. Although convergent validity may be lower in IQOL measures, content validity, on the contrary, is quite high. Content validity is the degree to which the content of an instrument is an adequate reflection of the construct to be measured [29]. IQOL measures have high content validity because the individual determines what constitutes his/her QOL [30]. Individualization of items 
maximizes content validity and guarantees their importance to every participant in a study.

The PGI domains nominated by patients in our sample were diverse and included dexterity, walking, sleep, fatigue, cognition, tremors, sports, depression, anxiety, self-care, speech, socializing, household tasks, bowel and bladder, work/employment, and balance. Although some of these domains are included in the PDQ-8, several are not. This may explain the moderate correlation observed between the two measures. We may have observed larger correlation values if the PGI had been compared with the more comprehensive version of the PDQ-8, the PDQ-39. Marinus and colleagues reported in a systematic review that the content between different PD-specific HRQL measures differed considerably [31]. The authors reported that although the PDQ-39 included most domains important to people with $\mathrm{PD}$, it lacked items on self-image, nighttime sleep problems, sexual activity, and transfers. Furthermore, our research team recently assessed the content validity of generic preference-based measures of HRQL (i.e., utility measures) such as the EQ-5D in PD [27]. These HRQL measures are typically used to evaluate the cost-effectiveness of different interventions. Our results showed that several domains important to people with PD were missing in these generic measures and that the development of a disease-specific preference-based measure may be warranted for use in costeffectiveness analysis in PD.

The magnitude of the correlation is also affected by the range of the variables understudy [32]. For example, putting the PGI, PFI, and PDQ- 8 , all on the same scale (0-100 with 100 best) and calculating confidence intervals, even the upper bound of the CI for the PGI (mean: 42; 95\% CI: 40-44) is well outside of the lower bound of the other measures (PDQ-8 transformed mean: 73; 95\% CI: 71-75; PFI mean: 64; 95\% CI: 60-67). Thus, there are more people at the lower end of the PGI than there are in the other 2 measures. In addition, the PGI usually captures only areas of concern whereas with other measures, the value is driven by not having deficits that are queried. These factors have been reported before [10], and thus, strong correlations are not expected.

For at least one item per PGI area, the PPV for the PGI was high. In other words, nominating an area was highly predictive of responding equivalently on a standardized item (see Table 4). For example, nominating walking as an area affecting quality of life was $80 \%$ predictive of being unable to walk more than a kilometer, and nominating fatigue (or depression) was $94 \%$ (86\%) predictive of rating fatigue (depression) as high. NPV values were not as high as expected because just because an area is not nominated does not mean the person does not have the problem, it is just not as important as those nominated.

Scores observed on the various PRO measures in our sample were similar to the literature. Jenkinson and Fitzpatrick reported on the PDQ-8 in 121 people with PD in Canada [33]. The average PDQ-8 score for their sample was 31.4 , which was similar to the average score of 27.0 in our sample. Both samples were similar in disease severity and duration. In addition, individuals who nominated walking as an area of concern on the PGI had similar PFI values to previous studies that have used this measure in PD [34-36]. People who did not nominate walking as an area of concern had a mean of $72 / 100$, which is similar to published normative data for the same age group (mean 75.7/100) [37]. For the PDQ-20, individuals who nominated memory or concentration as an area of concern had a mean value of $35 / 80$, which is close to the cutoff value of $\geq 40$ that is indicative of cognitive impairment [38]. Individuals who did not nominate this area as a concern had a considerably lower mean value of $22 / 80$ on the PDQ-20.

One of the advantages of IQOL measures is that they use a patient-centered approach. IQOL measures capture aspects of QOL that are most important to the individual. Unlike standard PRO measures, IQOL does not consist of predetermined domains. They allow the individual to nominate the domains that are important to the quality of his/her life. What further distinguishes IQOL from standard measures is that they allow the individual to determine the relative importance of each domain [8]. Standard measures assume that there is equal weighting between the domains measured. Another advantage of IQOL measures that is not commonly reported is their ability to serve as powerful evaluative instruments [8]. An evaluative measure is one that is responsive to changes in individuals or groups over time. Individualization of questionnaires can maximize responsiveness because (1) the domains are nominated by the patient (high content validity); (2) consequently, it facilitates the detection of the "signal" or true change; and (3) it minimizes and may eliminate floor and ceiling effects [30]. This study was cross-sectional, but future research should evaluate the responsiveness of the PGI in people with PD.

IQOL measures also have the potential to be used in clinical practice [8]. Standard PRO measures of QOL have often been difficult to use in clinical practice because the results are not easily interpretable by clinicians [39]. Clinicians at times face an uncertainty as to what the scores mean and how to apply the information [39]. On the other hand, IQOL measures may be simpler to use and interpret than standard PRO measures. The PGI can allow clinicians to quickly identify the areas that are of concern to the patient and that need to be addressed during treatment. Furthermore, scoring each domain from 0 to 10 allows them to assess the impact of the disease on each domain, and the distribution of the "points" among the domains determines the relative importance of each. A further advantage of IQOL instruments is that they can improve communication between the patient and the physician [40]. For rehabilitation professionals, IQOL measures can help prioritize treatment options by identifying the areas that the patient wants to improve the most. The domains generated by patients can be allowed to vary over time or they can be kept the same over time. In a clinical setting, clinicians may prefer to show patients the domains that were identified at baseline again at the follow-up visit to monitor how their patient's HRQL changes over time. All in all, clinicians need measures that are patient-centered, easy to use, clinically relevant, and sensitive to change-criteria that may be met by IQOL measures. 
A limitation of this study is the small sample size and that patients with PD were recruited from only one clinical site. Further research should be conducted on a larger sample size and longitudinal validity (i.e., responsiveness) of the PGI in PD should be tested.

\section{Conclusions}

In conclusion, the PGI is an IQOL that allows patients to nominate, rate, and value areas that have the most impact on their quality of life. This study demonstrated that nominated areas of QOL on the PGI provided comparable information to standard PRO measures, and presented evidence in support of the validity of personalized measures in PD.

\section{Data Availability}

The data used to support the findings of this study are restricted by the McGill University Health Center Research Ethics Board in order to protect patient privacy. Data are available from Nancy Mayo (nancy.mayo@mcgill.ca) for researchers who meet the criteria for access to confidential data.

\section{Conflicts of Interest}

The authors have no conflicts of interest to declare.

\section{Acknowledgments}

Dr. Kuspinar holds a 2019 Parkinson Canada New Investigator Award.

\section{Supplementary Materials}

Figure 2: distribution of the PDQ-8. Figure 3: distribution of the PGI. (Supplementary Materials)

\section{References}

[1] S. G. Brauer and M. E. Morris, "Can people with Parkinson's disease improve dual tasking when walking?" Gait \& Posture, vol. 31, no. 2, pp. 229-233, 2010.

[2] J. H. Friedman, R. G. Brown, C. Comella et al., "Fatigue in Parkinson's disease: a review," Movement Disorders, vol. 22, no. 3, pp. 297-308, 2007.

[3] D. Weintraub and E. Mamikonyan, "The neuropsychiatry of Parkinson disease: a perfect storm," The American Journal of Geriatric Psychiatry, vol. 27, 2019.

[4] C. P. Galts, L. E. Bettio, D. C. Jewett et al., "Depression in neurodegenerative diseases: common mechanisms and current treatment options," Neuroscience \& Biobehavioral Reviews, vol. 102, pp. 56-84, 2019.

[5] G. D. Kuhlman, J. L. Flanigan, S. A. Sperling, and M. J. Barrett, "Predictors of health-related quality of life in Parkinson's disease," Parkinsonism \& Related Disorders, vol. 65, pp. 86-90, 2019.

[6] C. R. B. Joyce, H. M. McGee, and C. A. O’Boyle, Individual Quality of Life: Approaches to Conceptualisation and Assessment, Taylor \& Francis, Didcot, UK, 1999.
[7] M. P. Dijkers, "Individualization in quality of life measurement: instruments and approaches," Archives of Physical Medicine and Rehabilitation, vol. 84, pp. S3-S14, 2003.

[8] C. O’Boyle, S. Höfer, and L. Ring, "Individualized quality of life," Assessing the Quality of Life in Clinical Trials, vol. 2, pp. 225-242, 2005.

[9] S. S. Tavernier, S. L. Beck, M. F. Clayton, M. A. Pett, and D. L. Berry, "Validity of the patient generated index as a quality-of-life measure in radiation oncology," Oncology Nursing Forum, vol. 38, 2011.

[10] N. E. Mayo, A. Aburub, M.-J. Brouillette et al., "In support of an individualized approach to assessing quality of life: comparison between patient generated index and standardized measures across four health conditions," Quality of Life Research, vol. 26, no. 3, pp. 601-609, 2017.

[11] D. A. Ruta, A. M. Garratt, M. Leng, I. T. Russell, and L. M. MacDonald, "A New approach to the measurement of quality of life," Medical Care, vol. 32, no. 11, pp. 1109-1126, 1994.

[12] I. Løchting, M. Grotle, K. Storheim, E. L. Werner, and A. M. Garratt, "Individualized quality of life in patients with low back pain: reliability and validity of the patient generated index," Journal of Rehabilitation Medicine, vol. 46, no. 8, pp. 781-787, 2014

[13] M. D. Witham, R. L. Fulton, L. Wilson, C. A. Leslie, and M. E. McMurdo, "Validation of an individualised quality of life measure in older day hospital patients," Health and Quality of Life Outcomes, vol. 6, no. 1, p. 27, 2008.

[14] K. K. Patel, D. L. Veenstra, and D. L. Patrick, "A review of selected patient-generated outcome measures and their application in clinical trials," Value in Health, vol. 6, no. 5, pp. 595-603, 2003.

[15] F. Martin, L. Camfield, K. Rodham, P. Kliempt, and D. Ruta, "Twelve years-experience with the patient generated index (PGI) of quality of life: a graded structured review," Quality of Life Research, vol. 16, no. 4, pp. 705-715, 2007.

[16] C. Jenkinson, R. Fitzpatrick, V. Peto, R. Greenhall, and N. Hyman, "The PDQ-8: development and validation of a short-form Parkinson's disease questionnaire," Psychology \& Health, vol. 12, no. 6, pp. 805-814, 1997.

[17] R. D. Hays and L. S. Morales, "The RAND-36 measure of health-related quality of life," Annals of Medicine, vol. 33, no. 5, pp. 350-357, 2001.

[18] K. Jongenelis, D. L. Gerritsen, A. M. Pot et al., "Construction and validation of a patient- and user-friendly nursing home version of the geriatric depression scale," International Journal of Geriatric Psychiatry, vol. 22, no. 9, pp. 837-842, 2007.

[19] M. Serrano-Dueñas, P. Martínez-Martín, T. Merchán, R. Bravo, and M. Serrano, "Properties of the apathy scale (AS) for use on Parkinson's patients," Advances in Parkinson's Disease, vol. 02, no. 02, pp. 53-57, 2013.

[20] R. A. Marrie, D. M. Miller, G. J. Chelune, and J. A. Cohen, "Validity and reliability of the MSQ LI in cognitively impaired patients with multiple sclerosis," Multiple Sclerosis Journal, vol. 9, no. 6, pp. 621-626, 2003.

[21] A. Rosenzveig, A. Kuspinar, S. S. Daskalopoulou, and N. E. Mayo, "Toward patient-centered care: a systematic review of how to ask questions that matter to patients," Medicine, vol. 93, no. 22, 2014.

[22] D. F. Cella and S. W. Perry, "Reliability and concurrent validity of three visual-analogue mood scales," Psychological Reports, vol. 59, no. 2, pp. 827-833, 1986. 
[23] J. Cohen, Statistical Power Analysis for the Behavioral Sciences, Routledge, Abingdon, UK, 2013.

[24] Network NCC, "Cancer-related fatigue. Clinical practice guidelines in oncology," Journal of the National Comprehensive Cancer Network, vol. 1, no. 3, p. 308, 2003.

[25] V. Mock, A. P. Abernethy, A. Atkinson et al., "Cancer-related fatigue clinical practice guidelines in oncology," Journal of the National Comprehensive Cancer Network, vol. 5, no. 10, pp. 1054-1078, 2007.

[26] A. Ala'S, B. Gagnon, A. Rodríguez, and N. E. Mayo, "Agreement between personally generated areas of quality of life concern and standard outcome measures in people with advanced cancer," Supportive Care in Cancer, vol. 24, no. 9, pp. 3831-3838, 2016.

[27] A. Kuspinar, K. Mate, A.-L. Lafontaine, and N. Mayo, "Evaluating the content validity of generic preference-based measures for use in Parkinson's disease," Parkinsonism \& Related Disorders, vol. 62, 2019.

[28] L. Wettergren, Å. Kettis-Lindblad, M. Sprangers, and L. Ring, "The use, feasibility and psychometric properties of an individualised quality-of-life instrument: a systematic review of the SEIQoL-DW," Quality of Life Research, vol. 18, no. 6, pp. 737-746, 2009.

[29] H. C. De Vet, C. B. Terwee, L. B. Mokkink, and D. L. Knol, Measurement in Medicine: A Practical Guide, Cambridge University Press, Cambridge, UK, 2011.

[30] Y. Lacasse, E. Wong, and G. H. Guyatt, Individualising Questionnaires. Individual Quality of Life: Approaches to Conceptualization and Assessment, pp. 87-103, Harwood Academic Publishers, Amsterdam, The Netherlands, 1999.

[31] J. Marinus, C. Ramaker, J. J. van Hilten, and A. M. Stiggelbout, "Health related quality of life in Parkinson's disease: a systematic review of disease specific instruments," Journal of Neurology, Neurosurgery \& Psychiatry, vol. 72, no. 2, pp. 241-248, 2002.

[32] L. D. Goodwin and N. L. Leech, "Understanding correlation: factors that affect the size of $\mathrm{r}$," The Journal of Experimental Education, vol. 74, no. 3, pp. 249-266, 2006.

[33] C. Jenkinson and R. Fitzpatrick, "Cross-cultural evaluation of the short form 8-item Parkinson's disease questionnaire (PDQ-8): results from America, Canada, Japan, Italy and Spain," Parkinsonism \& Related Disorders, vol. 13, no. 1, pp. 22-28, 2007.

[34] C. Jenkinson, V. Peto, R. Fitzpatrick, R. Greenhall, and N. Hyman, "Self-reported functioning and well-being in patients with Parkinson's disease: comparison of the shortform health survey (SF-36) and the Parkinson's Disease Questionnaire (PDQ-39)," Age and Ageing, vol. 24, no. 6, pp. 505-509, 1995.

[35] P. Hagell, A. L. Törnqvist, and J. Hobart, "Testing the SF-36 in Parkinson's disease," Journal of Neurology, vol. 255, no. 2, pp. 246-254, 2008.

[36] A. Schrag, M. Jahanshahi, and N. Quinn, "How does Parkinson's disease affect quality of life? A comparison with quality of life in the general population," Movement Disorders, vol. 15, no. 6, pp. 1112-1118, 2000.

[37] W. M. Hopman, T. Towheed, T. Anastassiades et al., "Canadian normative data for the SF-36 health survey," CMAJ, vol. 163 , no. 3, pp. 265-271, 2000.

[38] M. J. Sullivan, K. Edgley, and E. Dehoux, “A survey of multiple sclerosis: I. Perceived cognitive problems and compensatory strategy use," Canadian Journal of Rehabilitation, vol. 4, 1990.
[39] G. Velikova and P. Wright, "Individual patient monitoring," in Assessing Quality of Life in Clinical Trials, pp. 291-306, Oxford University Press, Oxford, UK, 2005.

[40] S. B. Detmar, M. J. Muller, J. H. Schornagel, L. D. V. Wever, and N. K. Aaronson, "Health-related quality-of-life assessments and patient-physician communication," Jama, vol. 288, no. 23, pp. 3027-3034, 2002. 